TRANSACTIONS OF THE

AMERICAN MATHEMATICAL SOCIETY

Volume 355, Number 3, Pages 967-985

S 0002-9947(02)03138-0

Article electronically published on November 1, 2002

\title{
NON-INDEPENDENCE OF EXCURSIONS OF THE BROWNIAN SHEET AND OF ADDITIVE BROWNIAN MOTION
}

\author{
ROBERT C. DALANG AND T. MOUNTFORD
}

\begin{abstract}
A classical and important property of Brownian motion is that given its zero set, distinct excursions away from zero are independent. In this paper, we examine the analogous question for the Brownian sheet, and also for additive Brownian motion. Our main result is that given the level set of the Brownian sheet at level zero, distinct excursions of the sheet away from zero are not independent. In fact, given the zero set of the Brownian sheet in the entire non-negative quadrant, and the sign of all but a finite number of excursions away from zero, the signs of the remaining excursions are determined. For additive Brownian motion, we prove the following definitive result: given the zero set of additive Brownian motion and the sign of a single excursion, the signs of all other excursions are determined.

In an appendix by John B. Walsh, it is shown that given the absolute value of the sheet in the entire quadrant and, in addition, the sign of the sheet at a fixed, non-random time point, then the whole sheet can be recovered.
\end{abstract}

\section{INTRODUCTION}

The (standard) Brownian sheet is a centered, continuous Gaussian process $W=$ $\left(W(t), t \in \mathbb{R}_{+}^{2}\right)$, defined on a complete probability space $(\Omega, \mathcal{F}, P)$ and indexed by the positive quadrant in the plane, with covariance given by

$$
E\left(W\left(s_{1}, s_{2}\right) W\left(t_{1}, t_{2}\right)\right)=\left(s_{1} \wedge t_{1}\right)\left(s_{2} \wedge t_{2}\right) .
$$

It is one of the natural extensions of Brownian motion to higher-dimensional time. Many of its properties are straightforward analogues of properties of Brownian motion, but in this paper, we shall demonstrate a dramatic qualitative difference between Brownian motion and the Brownian sheet.

We consider Brownian q-bubbles (or in this paper simply q-bubbles), the components of the random time set

$$
\left\{\left(t_{1}, t_{2}\right): W\left(t_{1}, t_{2}\right) \neq q\right\} .
$$

$L_{+}(q)$ (resp. $\left.L_{-}(q)\right)$ will denote the set of $\left(t_{1}, t_{2}\right)$ such that $W\left(t_{1}, t_{2}\right)>q$ (resp. $\left.W\left(t_{1}, t_{2}\right)<q\right)$, while $L(q)=\left\{\left(t_{1}, t_{2}\right): W\left(t_{1}, t_{2}\right)=q\right\}$ will simply denote the level

Received by the editors November 5, 2001 and, in revised form, June 21, 2002.

2000 Mathematics Subject Classification. Primary 60G60; Secondary 60G17, 60G15.

Key words and phrases. Brownian sheet, excursions, level sets, additive Brownian motion.

The research of the first author is partially supported by the Swiss National Foundation for Scientific Research.

The research of the second author was partially supported by NSF grant DMS-00-71471 and by the BRIMS Institute (Bristol). 
set at level $q$. Components of $L_{+}(q)$ will be called upward bubbles, while those of $L_{-}(q)$ will be refered to as downward bubbles. When $(q)$ is absent, $L_{+}, L_{-}$and $L$ refer to the case $q=0$. In this case, we may simply refer to bubbles, and a bubble is said to be positive if $W$ is positive on it, negative otherwise.

By path continuity of the sheet, bubbles are necessarily open sets. For the sheet, positive and negative bubbles are natural analogues of positive and negative excursions of Brownian motion $B$ away from zero. It is a much celebrated fact (see e.g. 4], 8], 9] that given $\sigma(|B(t)|, t \geq 0$ ), the signs of the excursions of $B$ away from 0 are independent and symmetric; this remains true if $\sigma(|B(t)|, t \geq 0)$ is replaced by the $\sigma$-field generated by the zero set of $B$. It is further known that given the zero set of a Brownian motion, the laws of the respective excursions are independent, with a law that is well-known (see above references). It seems difficult to develop a theory for bubbles along the same lines. In great part, this is because, as we show in this paper, the natural analogue to the first phenomenon does not hold for the sheet: given the absolute value of the sheet, or even only $L(0)$, the signs of the bubbles are highly dependent.

Definition 1.1. Consider a given sample path of $W$.

(a) For $q \in \mathbb{R}$, if $C$ and $\tilde{C}$ are distinct $q$-bubbles for which there is a point $\left(s_{1}, s_{2}\right) \in \mathbb{R}_{+}^{2}$ such that $\left(s_{1}, s_{2}-v\right) \in C$ and $\left(s_{1}+v, s_{2}\right) \in \tilde{C}$ for all sufficiently small $v>0$, we say that $C$ and $\tilde{C}$ are hinged. We also say that $C$ and $\tilde{C}$ are hinged if, in the above, we replace $\left(s_{1}, s_{2}-v\right)$ with $\left(s_{1}, s_{2}+v\right)$ or $\left(s_{1}+v, s_{2}\right)$ with $\left(s_{1}-v, s_{2}\right)$.

(b) If, in addition, there is $\alpha>0$ such that $\left|W\left(s_{1}, s_{2}-v\right)-W\left(s_{1}, s_{2}\right)\right|>v^{\alpha}$ and $\left|W\left(s_{1}+v, s_{2}\right)-W\left(s_{1}, s_{2}\right)\right|>v^{\alpha}$ for all sufficiently small $v>0$, we say that the growth exponent of $W$ at $\left(s_{1}, s_{2}\right)$ is $\leq \alpha$. We say that the distance exponent at $\left(s_{1}, s_{2}\right)$ is $\leq \beta$ if for all sufficiently small $v>0, d\left(\left(s_{1}, s_{2}-v\right), L(q)\right) \geq v^{\beta}$ and $d\left(\left(s_{1}+v, s_{2}\right), L(q)\right) \geq v^{\beta}$, where $d(s, A)$ denotes the infimum over $t \in A$ of the distance $d(s, t)$.

Theorem 1. With probability one, there is no $q \in \mathbb{R}$ and a distinct pair of $q$-bubbles of the same direction that are hinged.

Theorem 2. Let $C(1,1)$ be the 0-bubble containing $(1,1)$. With probability one, $C(1,1)$ is hinged to infinitely many other 0-bubbles (necessarily of opposite direction by Theorem 11). For $\alpha>\frac{1}{2}$, these bubbles can be chosen so that the growth exponent at the corner of the hinge is $\leq \alpha$, or so that the distance exponent at the corner of the hinge is $\leq 2 \alpha$.

Theorem [2, which extends a result of [1. Theorem 1], has the following interesting consequence. Given the $\sigma$-field $\mathcal{G}$ generated by $L(=L(0))$ and all null sets (throughout the paper, all sigma-fields will be assumed to be complete with respect to the relevant probability measure, even though this will not be explicitly mentioned), we can ask whether the signs of these bubbles are conditionally independent. A consequence of Theorem 2 is that the answer to this question is negative. We make this statement precise as follows.

Set

$$
\begin{aligned}
\mathcal{G} & =\sigma\{d(x, L), x \text { with rational coordinates }\}=\sigma\left\{d(x, L), x \in \mathbb{R}_{+}^{2}\right\} \\
& =\sigma\left\{I_{L \cap D \neq \emptyset}, D \text { a square in } \mathbb{R}_{+}^{2}\right\} .
\end{aligned}
$$

Let $E_{1}, E_{2}, \ldots$ be an enumeration of the 0-bubbles that is measurable with respect to the $\sigma$-field $\mathcal{G}$. Such an enumeration can be obtained as follows. Let 
$\left(q_{i}, i \in \mathbb{N}\right)$ be an enumeration of the points in $] 0, \infty\left[{ }^{2}\right.$ with rational coordinates. Let $E_{1}$ be the bubble containing $q_{1}$, and for $i>1$, let $E_{i}$ be the bubble containing $q_{J_{i}}$, where

$$
J_{i}=\inf \left\{k \geq 1: q_{k} \notin \bigcup_{\ell=1}^{i-1} E_{\ell} \text { and } W\left(q_{k}\right) \neq 0\right\} .
$$

Given such an enumeration of bubbles, define random variables $S_{i}$ with values in $\{-1,1\}$ such that

that is, $S_{i}$ is the sign of $W$ on $E_{i}$.

$$
S_{i} W(t)>0 \quad \text { for } t \in E_{i},
$$

Theorem 3. For $n \in \mathbb{N}$, let $\mathcal{F}_{n}=\mathcal{G} \vee \sigma\left\{S_{i}, i>n\right\}$. Then for all $n \geq 0$,

$$
\mathcal{F}_{n}=\mathcal{F}_{0} .
$$

In other words, given the level set $L(0)$ and the sign of all but finitely many bubbles, one can determine the signs of the remaining bubbles.

A related result, where we are given the absolute value of the Brownian sheet rather than the level set $L(0)$ and which is due to John B. Walsh, is given in the Appendix.

Locally (with time suitably rescaled), the Brownian sheet $W$ closely resembles additive Brownian motion, that is, the process $X=\left(X\left(s_{1}, s_{2}\right),\left(s_{1}, s_{2}\right) \in \mathbb{R}^{2}\right)$ defined by $X\left(s_{1}, s_{2}\right)=B_{1}\left(s_{1}\right)-B_{2}\left(s_{2}\right)$, where $B_{1}$ and $B_{2}$ are independent (twosided) Brownian motions. Thus the following strong result for the process $X$ is of interest.

Theorem 4. For the additive Brownian motion process $X$,

$$
\mathcal{G}^{\prime} \vee \sigma\left\{S_{1}^{\prime}\right\}=\mathcal{F}_{0}^{\prime},
$$

where $\mathcal{G}^{\prime}, S_{1}^{\prime}$ and $\mathcal{F}_{0}^{\prime}$ are the natural analogues for the process $X$ of the objects $\mathcal{G}$, $S_{1}$ and $\mathcal{F}_{0}$ defined for $W$. In other words, given the level set of $X$ at level 0 and the sign of a single excursion, the signs of all the other excursions are determined.

\section{Proof of Theorems 1, 2 and 3}

We begin by proving Theorem 3 assuming Theorems 1 and 2

Proof of Theorem 3. We first examine the measurability properties of the bubbles $E_{i}$. In the following, a path $\gamma$ will be a continuous function from $[0,1]$ to $\mathbb{R}^{2}$. If $\gamma(0)=s$ and $\gamma(1)=t$, we say that $\gamma$ is a path from $s$ to $t$.

For $s, t \in \mathbb{R}_{+}^{2}$, let $A(s, t)$ be the event " $s$ and $t$ are in the same bubble." We first show that $A(s, t) \in \mathcal{G}$. Since an open set in $\mathbb{R}^{2}$ is connected if and only if it is path connected,

$$
A(s, t)=\{\omega \in \Omega: \text { there exists a path } \gamma \text { from } s \text { to } t \text { on which } W \neq 0\} .
$$

For $m \in \mathbb{N}$, set

$$
\mathbb{D}_{m}=\left\{\left(i 2^{-m}, j 2^{-m}\right): i, j \in \mathbb{N}\right\} .
$$

For $k, m \in \mathbb{N}$, let $\Gamma_{k}^{m}(s, t)$ be the set of paths $\gamma$ from $s$ to $t$ such that for all $i \in\{0, \ldots, k\}$,

(i) $\gamma\left(\frac{i}{k}\right) \in \mathbb{D}_{m} \cup\{s\} \cup\{t\}$,

(ii) $\left|\gamma\left(\frac{i}{k}\right)-\gamma\left(\frac{i-1}{k}\right)\right| \leq 2^{-m}$, 
(iii) $\gamma$ is linear on $\left[\frac{i-1}{k}, \frac{i}{k}\right]$.

We note that the set $\Gamma_{k}^{m}(s, t)$ is finite. Let $\mathbb{Q}$ denote the set of rational numbers. It is not difficult to check that

$$
A(s, t)=\bigcup_{n=1}^{\infty} \bigcup_{m=1}^{\infty} \bigcup_{k=1}^{\infty} \bigcup_{\gamma \in \Gamma_{k}^{m}(s, t)} \bigcap_{r \in \mathbb{Q} \cap[0,1]}\left\{d(\gamma(r), L) \geq \frac{1}{n}\right\}
$$

and the set on the right-hand side clearly belongs to $\mathcal{G}$.

It is straightforward to deduce from this that $E_{i}$ is measurable with respect to $\mathcal{G}$, in the sense that for all $i \geq 1$ and $t \in \mathbb{R}_{+}^{2},\left\{t \in E_{i}\right\} \in \mathcal{G}$.

Fix $\beta>1$ and, for $n \geq 1$, define

$$
\begin{aligned}
C^{n}(i, j)=\left\{\exists\left(s_{1}, s_{2}\right) \in \mathbb{R}_{+}^{2}:\left(s_{1}, s_{2}-v\right) \in E_{i},\left(s_{1}+v, s_{2}\right) \in E_{j},\right. \\
\left.\quad d\left(\left(s_{1}, s_{2}-v\right), L\right) \geq v^{\beta} \text { and } d\left(\left(s_{1}+v, s_{2}\right), L\right) \geq v^{\beta} \text { for } 0 \leq v \leq \frac{1}{n}\right\}
\end{aligned}
$$

and

$$
C(i, j)=\bigcup_{n=1}^{\infty} C^{n}(i, j)
$$

Then $C(i, j)$ is the event "bubbles $E_{i}$ and $E_{j}$ are hinged, with distance exponent $\leq \beta$." We claim that $C(i, j) \in \mathcal{G}$. To prove this, it suffices to show that $C^{n}(i, j) \in \mathcal{G}$, for all $n \geq 1$. Letting $\mathbb{D}_{m}(R)=\mathbb{D}_{m} \cap[0, R]^{2}$, this follows from the equality

$$
\begin{gathered}
C^{n}(i, j)=\bigcup_{R=1}^{\infty} \bigcap_{\ell=1}^{\infty} \bigcup_{m=1}^{\infty} \bigcup_{s \in \mathbb{D}_{m}(R)}\left\{d(s, L) \leq 1 / \ell,\left(s_{1}, s_{2}-\frac{1}{n}\right) \in E_{i},\right. \\
d\left(\left(s_{1}, s_{2}-v\right), L\right) \geq v^{\beta}-1 / \ell,\left(s_{1}+\frac{1}{n}, s_{2}\right) \in E_{j}, \\
\left.d\left(\left(s_{1}+v, s_{2}\right), L\right) \geq v^{\beta}-1 / \ell, 0 \leq v \leq 1 / n\right\} .
\end{gathered}
$$

We now prove the statement in Theorem 3, Fix $n>0$. Clearly, $\mathcal{F}_{n} \subset \mathcal{F}_{0}$, and so we only need to show that $S_{i}$ is $\mathcal{F}_{n}$-measurable, for $n \geq 1$ and $i=1, \ldots, n$. In fact, by Theorems 1 and 2 a.s.

$$
S_{i}=\left\{\begin{aligned}
1 & \text { on } \limsup _{j \rightarrow \infty}\left(\left\{S_{j}=-1\right\} \cap C(i, j)\right), \\
-1 & \text { on } \limsup _{j \rightarrow \infty}\left(\left\{S_{j}=1\right\} \cap C(i, j)\right),
\end{aligned}\right.
$$

and the right-hand side is $\mathcal{F}_{n}$-measurable by the definition of $\mathcal{F}_{n}$ and the fact that $C(i, j) \in \mathcal{G}$. This proves Theorem 3 .

Proof of Theorem 1. It suffices to prove the statement of the theorem for upward bubbles. Observe that if $\left.] t_{1}, t_{1}+h\right] \times\left\{t_{2}\right\}$ and $\left.\left\{t_{1}\right\} \times\right] t_{2}, t_{2}+h[$ do belong to distinct components of $L_{+}(q)$, then $W\left(t_{1}, t_{2}\right)$ must equal $q$. In view of scaling properties of the Brownian sheet, it is sufficient to show that for fixed $h$, the event "there does not exist $\left(t_{1}, t_{2}\right) \in[1,3 / 2]^{2}$ such that properties (i), (ii) and (iii) below hold" has probability 0 , where properties (i), (ii) and (iii) are as follows:

(i) $W\left(t_{1}+u, t_{2}\right)>W\left(t_{1}, t_{2}\right), 0<u \leq h$,

(ii) $W\left(t_{1}, t_{2}+v\right)>W\left(t_{1}, t_{2}\right), 0<v \leq h$,

(iii) $] t_{1}, t_{1}+h\left[\times\left\{t_{2}\right\}\right.$ and $\left.\left\{t_{1}\right\} \times\right] t_{2}, t_{2}+h[$ belong to distinct components of $L_{+}\left(W\left(t_{1}, t_{2}\right)\right)$.

In the following we take $h=1$. It will be clear that the proof carries over to any fixed positive $h$. 
For $s=\left(s_{1}, s_{2}\right) \in \mathbb{D}_{2 n}$, let $F(s, n)$ be the event just described but with the additional requirement

$$
\left(t_{1}, t_{2}\right) \in\left[s_{1}, s_{1}+2^{-2 n}\right] \times\left[s_{2}, s_{2}+2^{-2 n}\right] .
$$

It suffices to show that $P\left(\bigcup_{s \in \mathbb{D}_{2 n}} F(s, n)\right)=0$, which will certainly follow from the asymptotic property $\sup _{s \in \mathbb{D}_{2 n}} P(F(s, n))=o\left(2^{-4 n}\right)$.

Let

$$
W_{R}^{s}(u)=W\left(s_{1}+u, s_{2}\right)-W\left(s_{1}, s_{2}\right), \quad W_{U}^{s}(v)=W\left(s_{1}, s_{2}+v\right)-W\left(s_{1}, s_{2}\right),
$$

and define

$$
\begin{aligned}
& G_{0}(s, n)=\left\{\sup _{\left(u_{1}, u_{2}\right) \in\left[s_{1}, s_{1}+2^{-2 n}\right] \times\left[s_{2}, s_{2}+2^{-2 n}\right]}\left|W\left(u_{1}, u_{2}\right)-W\left(s_{1}, s_{2}\right)\right|<n 2^{-n}\right\}, \\
& G_{R}(s, n)=\left\{W_{R}^{s}(u)>-n 2^{-n}, 0 \leq u \leq \frac{1}{2}\right\}, \\
& G_{U}(s, n)=\left\{W_{U}^{s}(v)>-n 2^{-n}, 0 \leq v \leq \frac{1}{2}\right\}, \\
& G(s, n)=G_{0}(s, n) \cap G_{R}(s, n) \cap G_{U}(s, n) .
\end{aligned}
$$

We show first that $P(F(s, n) \backslash G(s, n)) \leq K e^{-n^{2} / 8}$. Indeed, $F(s, n) \backslash G(s, n)$ is contained in the union of the three events

$$
\begin{gathered}
\left\{\sup _{\left(u_{1}, u_{2}\right) \in\left[s_{1}, s_{1}+2^{-2 n}\right] \times\left[s_{2}, s_{2}+2^{-2 n}\right]}\left|W\left(u_{1}, u_{2}\right)-W\left(s_{1}, s_{2}\right)\right|>n 2^{-n}\right\}, \\
\left\{\sup _{1 \leq u \leq 2} \sup _{0 \leq v \leq 2^{-2 n}}\left|W\left(u, s_{2}+v\right)-W\left(u, s_{2}\right)\right|>n 2^{-n}\right\}
\end{gathered}
$$

and

$$
\left\{\sup _{0 \leq u \leq 2^{-2 n}} \sup _{1 \leq v \leq 2}\left|W\left(s_{1}+u, v\right)-W\left(s_{1}, v\right)\right|>n 2^{-n}\right\} .
$$

By the scaling properties of the Brownian sheet, the probability of these last two events is equal to

$$
P\left(\left\{\sup _{1 \leq u \leq 2} \sup _{0 \leq v \leq 1}|W(u, v)|>n\right\}\right),
$$

and according to [7. Lemma 1.2], this probability is bounded by $4 e^{-n^{2} / 8}$ for large $n$. By [7, there is $c>0$ such that the probability of the first event is $\leq c e^{-n^{2} / 8}$.

Now let $\tau_{R}$ (resp. $\tau_{U}$ ) be the first time $W_{R}^{s}\left(\right.$ resp. $\left.W_{U}^{s}\right)$ hits level $n^{2} 2^{-n+1}$. Then

$$
\begin{aligned}
& P(F(s, n) \cap G(s, n)) \\
& \quad \leq P\left(G(s, n) \cap\left(\left\{\tau_{R} \vee \tau_{U}>n^{6} 2^{-2 n}\right\} \cup\left\{\tau_{R} \wedge \tau_{U}<2^{-2 n}\right\}\right)\right)+P(H(s, n)),
\end{aligned}
$$

where

$$
H(s, n)=F(s, n) \cap G(s, n) \cap\left\{2^{-2 n}<\tau_{R} \wedge \tau_{U} \leq \tau_{R} \vee \tau_{U}<n^{6} 2^{-2 n}\right\} .
$$

Clearly,

$$
\begin{aligned}
& P\left(G_{R}(s, n) \cap\left(\left\{\tau_{R}>n^{6} 2^{-2 n}\right\} \cup\left\{\tau_{R}<2^{-2 n}\right\}\right)\right) \\
& \quad \leq P\left(G_{R}(s, n) \cap\left\{\tau_{R}>n^{6} 2^{-2 n}\right\}\right)+P\left\{\tau_{R}<2^{-2 n}\right\} .
\end{aligned}
$$

Classical properties of the times a Brownian motion takes to leave an interval (see e.g. [5, Chap. VI, Lemma 8.1]) show that there are positive constants $c_{1}$ and $c_{2}$ such that the first term on the right-hand side is bounded above by

$$
c_{1} \exp \left(-c_{2} n^{2}\right) \text {, }
$$


and the same bound holds for $P\left\{\tau_{R}<2^{-2 n}\right\}$. Therefore, the first term in (1) is $\leq c \exp \left(-c n^{2}\right)$. As for the second term in (1), notice that for large $n$, on $H(s, n)$,

$$
\begin{aligned}
W\left(s_{1}+\tau_{R}, s_{2}+v\right) & =W(s)+W_{R}^{s}\left(\tau_{R}\right)+W_{U}^{s}(v)+Z(v) \\
& \geq W(s)+n^{2} 2^{-n+1}-n 2^{-n}+Z(v),
\end{aligned}
$$

where $\left.\left.\left.Z(v)=\Delta_{\text {] }} s_{1}, s_{1}+\tau_{R}\right] \times\right] s_{2}, s_{2}+v\right]$, and similarly,

$$
W\left(s_{1}+u, s_{2}+\tau_{U}\right) \geq W(s)+n^{2} 2^{-n+1}-n 2^{-n}+Z^{\prime}(u),
$$

where $Z^{\prime}(u)=\Delta_{\left.\left.\left.s_{1}, s_{1}+u\right] \times\right] s_{2}, s_{2}+\tau_{U}\right]} W$ (we have used here the notation

$$
\Delta_{] s_{1}, u_{1}\right] \times\right] s_{2}, u_{2}\right]} W=W\left(u_{1}, u_{2}\right)-W\left(s_{1}, u_{2}\right)-W\left(u_{1}, s_{2}\right)+W\left(s_{1}, s_{2}\right),
$$

if $s_{1} \leq u_{1}$ and $\left.s_{2} \leq u_{2}\right)$. For the particular $t=\left(t_{1}, t_{2}\right)$ satisfying (i), (ii) and (iii) above, $W(\cdot)-W(t)$ must hit 0 on the union of the two segments $\left\{s_{1}+\tau_{R}\right\} \times\left[s_{2}, s_{2}+\right.$ $\left.\tau_{U}\right]$ and $\left[s_{1}, s_{1}+\tau_{R}\right] \times\left\{s_{2}+\tau_{U}\right\}$. So for large $n$, on $H(s, n), W(\cdot)-W(s)$ must hit $n 2^{-n}$ on the union of these two segments. Therefore, on $H(s, n)$,

$$
\min \left(\inf _{0 \leq v \leq n^{6} 2^{-2 n}} Z(v), \inf _{0 \leq u \leq n^{6} 2^{-2 n}} Z^{\prime}(u)\right) \leq-n^{2} 2^{-n-1} .
$$

By the scaling properties of the Brownian sheet, the probability of this event is less than the following, for all large $n$ :

$$
2 P\left\{\sup _{0 \leq u \leq 1} B(u) \geq n^{-4} 2^{n}\right\} \leq c \exp \left(-n^{-8} 2^{2 n}\right) .
$$

This completes the proof of Theorem 1.

Remark 5. It is not difficult to check that the proof of Theorem 1 extends to the Brownian sheet with more than two parameters. Regarding Theorem 2, the statement for the two-parameter Brownian sheet implies the analogous statement for the multiparameter Brownian sheet.

We now turn to the proof of Theorem 2 which relies on several preliminaries and lemmas. As is usual, for points $s=\left(s_{1}, s_{2}\right)$ and $t=\left(t_{1}, t_{2}\right)$, we write $s \leq t$ (or equivalently, $t \geq s$ ) if $s_{i} \leq t_{i}$ for $i=1,2$.

Let $\tilde{\Omega}=C_{0}\left(\mathbb{R}_{+}^{2}, \mathbb{R}\right)$ be the set of continuous functions $\tilde{\omega}: \mathbb{R}_{+}^{2} \rightarrow \mathbb{R}$ such that $\tilde{\omega}(0,0)=0$ (but $\tilde{\omega}$ need not vanish elsewhere on the axes). Let $(Y(u, v), \quad(u, v) \in$ $\mathbb{R}_{+}^{2}$ ) be the canonical coordinate process

$$
Y(u, v)(\tilde{\omega})=\tilde{\omega}(u, v), \quad \tilde{\omega} \in \tilde{\Omega},
$$

and let $\tilde{\mathcal{B}}=\sigma\left(Y(u, v),(u, v) \in \mathbb{R}_{+}^{2}\right)$. We define two one-parameter filtrations $\left(\tilde{\mathcal{F}}_{u}^{1}, u \geq 0\right)$ and $\left(\tilde{\mathcal{F}}_{v}^{2}, v \geq 0\right)$ and a two-parameter filtration $\left(\tilde{\mathcal{F}}_{u, v},(u, v) \in \mathbb{R}_{+}^{2}\right)$ by

$$
\begin{gathered}
\tilde{\mathcal{F}}_{u}^{1}=\sigma\left\{Y\left(s_{1}, s_{2}\right): 0 \leq s_{1} \leq u, 0 \leq s_{2}\right\}, \\
\tilde{\mathcal{F}}_{v}^{2}=\sigma\left\{Y\left(s_{1}, s_{2}\right): 0 \leq s_{1}, 0 \leq s_{2} \leq v\right\},
\end{gathered}
$$

and

$$
\tilde{\mathcal{F}}_{u, v}=\sigma\left\{Y\left(s_{1}, s_{2}\right): 0 \leq s_{1} \leq u, 0 \leq s_{2} \leq v\right\}
$$

Recall that a stopping point relative to $\left(\tilde{\mathcal{F}}_{u, v}\right)$ is a random variable $T$ with values in $\mathbb{R}_{+}^{2}$ such that $\{T \leq(u, v)\} \in \tilde{\mathcal{F}}_{u, v}$, for all $(u, v) \in \mathbb{R}_{+}^{2}$.

Let

$$
\tilde{\tau}_{\varepsilon}=\inf \{v>0: Y(0, v)=\varepsilon\}
$$


and let $\tilde{C}_{\varepsilon}(\tilde{\omega})$ be the connected component of $\left\{(u, v) \in \mathbb{R}_{+}^{2}: Y(u, v)(\tilde{\omega})>0\right\}$ that contains $\left(0, \tilde{\tau}_{\varepsilon}(\tilde{\omega})\right)$.

Fix $\alpha>\frac{1}{2}$. Let $\tilde{G}_{\varepsilon}$ be the event "there exists $\left(u_{0}, v_{0}\right) \in\left[0, \varepsilon^{2}\right] \times\left[\tilde{\tau}_{\varepsilon}, \tilde{\tau}_{\varepsilon}+\varepsilon^{2}\right]$ such that $Y>0$ on $\left(\left[0, u_{0}\right] \times\left\{\tilde{\tau}_{\varepsilon}\right\}\right) \cup\left(\left\{u_{0}\right\} \times\left[\tilde{\tau}_{\varepsilon}, v_{0}[)\right.\right.$ and there is $\delta_{1}>0$ such that $Y\left(u_{0}, v_{0}-u\right)>u^{\alpha}$ and $Y\left(u_{0}+u, v_{0}\right)<-u^{\alpha}$ for $0<u<\delta_{1}$." Finally, set

$$
\tilde{F}=\limsup _{n \rightarrow \infty} \tilde{G}_{1 / n} \text {. }
$$

Notice that the events $\tilde{G}_{\varepsilon}$ and $\tilde{F}$ have been defined without reference to a probability measure on $(\tilde{\Omega}, \tilde{\mathcal{B}})$. We now define a family of such probability measures.

For $t=\left(t_{1}, t_{2}\right) \in \mathbb{R}_{+}^{2}$, let $P_{t}$ be the probability measure on $(\tilde{\Omega}, \tilde{\mathcal{B}})$ under which $Y$ has the same law as the process

$$
(u, v) \mapsto W\left(t_{1}+u, t_{2}+v\right)-W\left(t_{1}, t_{2}\right) .
$$

We note that under $P_{t}$, the law of $Y$ is identical to that of

$$
\sqrt{t_{2}} B_{1}(u)+\sqrt{t_{1}} B_{2}(v)+\hat{W}(u, v),
$$

where $B_{1}, B_{2}$ and $\hat{W}$ are independent, $B_{1}$ and $B_{2}$ are standard Brownian motions and $\hat{W}$ is a standard Brownian sheet.

The following zero-one law follows from the usual classical argument 7], given the independent increments property of $Y$ and the continuity of $Y$ under $P_{t}$.

Lemma 6. Under $P_{t}$, every event in the $\sigma$-field $\bigcap_{s_{1}, s_{2}>0} \sigma\{Y(u, v):(u, v) \leq$ $\left.\left(s_{1}, s_{2}\right)\right\}$ has probability 0 or 1 .

Lemma 7. (a) There is a constant $c_{0}>0$ such that, for all $0<\varepsilon<1$ and $t \geq(1,1), P_{t}\left(\tilde{G}_{\varepsilon}\right) \geq c_{0}$.

(b) For all $t \geq(1,1), P_{t}(\tilde{F})=1$.

Proof. Fix $0<\varepsilon<1$ and $t=\left(t_{1}, t_{2}\right) \geq(1,1)$.

(a) Let $V_{\varepsilon}=t_{2}+\tilde{\tau}_{\varepsilon}$. For $i=1, \ldots, 2^{2 n}$, set $x_{i}=i \varepsilon^{2} 2^{-2 n}$ and

$$
\mu_{i}=\inf \left\{v \geq 0: Y\left(x_{i} / V_{\varepsilon}, \tilde{\tau}_{\varepsilon}+v / t_{1}\right)=0\right\} .
$$

Define the event

$$
F_{t}(i, n)=F_{0} \cap F_{1}(i, n) \cap F_{2}(i, n) \cap F_{3}(i, n),
$$

where

$$
\begin{aligned}
F_{0} & =\left\{Y\left(u / V_{\varepsilon}, \tilde{\tau}_{\varepsilon}\right)>\varepsilon / 2, \text { for } 0 \leq u \leq \varepsilon^{2}\right\}, \\
F_{1}(i, n) & =\left\{\mu_{i} \leq \varepsilon^{2}\right\}, \\
F_{2}(i, n) & =\left\{Y\left(x_{i} / V_{\varepsilon}, \tilde{\tau}_{\varepsilon}+\left(\mu_{i}-v\right) / t_{1}\right) \geq v^{\alpha}-2^{-n}, \text { for } 0 \leq v \leq \mu_{i}\right\}, \\
F_{3}(i, n) & =\left\{Y\left(\left(x_{i}+u\right) / V_{\varepsilon}, \tilde{\tau}_{\varepsilon}+\mu_{i} / t_{1}\right) \leq-\varepsilon^{1-2 \alpha} u^{\alpha}+2^{-n}, \text { for } 0 \leq u \leq \varepsilon^{2}\right\} .
\end{aligned}
$$

Notice that $x_{i} / V_{\varepsilon}$ is $\tilde{\mathcal{F}}_{0}^{1}$-measurable, and therefore is a stopping time relative to $\left(\tilde{\mathcal{F}}_{u}^{1}\right)$. Because $H_{i}=\left(x_{i} / V_{\varepsilon}, \tilde{\tau}_{\varepsilon}+\mu_{i} / t_{1}\right)$ is a stopping point relative to $\left(\tilde{\mathcal{F}}_{u, v}\right)$ and $Y\left(H_{i}\right)=0$, conditionally given $\tilde{\mathcal{F}}_{x_{i} / V_{\varepsilon}}^{1}$,

$$
u \mapsto Y\left(\left(x_{i}+u\right) / V_{\varepsilon}, \tilde{\tau}_{\varepsilon}+\mu_{i} / t_{1}\right)
$$


is a Brownian motion started at 0 with speed $\left(V_{\varepsilon}+\mu_{i} / t_{1}\right)^{\frac{1}{2}} / V_{\varepsilon}^{\frac{1}{2}}$. On $F_{1}(i, n)$, this speed is in $\left[1,1+\varepsilon^{2}\right] \subset[1,2]$. By the scaling properties of Brownian motion, on $F_{1}(i, n)$,

$$
P_{t}\left(F_{3}(i, n) \mid \tilde{\mathcal{F}}_{x_{i} / V_{\varepsilon}}^{1}\right) \geq P\left\{B(u) \leq-u^{\alpha}+2^{-n} / \varepsilon, 0 \leq u \leq 1\right\} .
$$

Therefore, according to [1, Lemma 12], there is a universal constant $c_{1}>0$ such that

$$
P_{t}\left(F_{3}(i, n) \mid \tilde{\mathcal{F}}_{x_{i} / V_{\varepsilon}}^{1}\right) \geq c_{1} 2^{-n} / \varepsilon \quad \text { on } F_{1}(i, n)
$$

Notice that the process

$$
v \mapsto Y\left(x_{i} / V_{\varepsilon}, \tilde{\tau}_{\varepsilon}+\left(\mu_{i}-v\right) / t_{1}\right), \quad 0 \leq v \leq \mu_{i},
$$

is $\tilde{\mathcal{F}}_{x_{i} / V_{\varepsilon}}^{1}$-measurable, and has the law of a $\operatorname{Bessel}(3)$ process with speed

$$
\left(t_{1}+x_{i} / V_{\varepsilon}\right)^{\frac{1}{2}} / t_{1}^{\frac{1}{2}}
$$

run until it first hits an independent $N\left(\varepsilon, i \varepsilon^{2} 2^{-2 n}\right)$ random variable, which is $\geq \varepsilon / 2$ on $F_{0}$. This speed is in the interval $\left[1,1+\varepsilon^{2}\right] \subset[1,2]$. Because $v \mapsto v^{\alpha}$ is a lower escape function for the $\operatorname{Bessel}(3)$ process 6 Example 5.4.7], on $F_{0}$,

$$
P_{t}\left(F_{1}(i, n) \cap F_{2}(i, n) \mid \tilde{\mathcal{F}}_{\tilde{\tau}_{\varepsilon}}^{2}\right) \geq c_{2}
$$

where $c_{2}>0$ is a universal constant. Finally, because $\left(0, \tilde{\tau}_{\varepsilon}\right)$ is a stopping point relative to $\left(\tilde{\mathcal{F}}_{u, v}\right)$,

$$
u \mapsto Y\left(u / V_{\varepsilon}, \tilde{\tau}_{\varepsilon}\right)-Y\left(0, \tilde{\tau}_{\varepsilon}\right)
$$

is a standard Brownian motion; so $P_{t}\left(F_{0}\right) \geq c_{3}$, where $c_{3}>0$ is a universal constant. Setting $c=c_{1} c_{2} c_{3}$, we conclude that

$$
P_{t}\left(F_{t}(i, n)\right) \geq c 2^{-n} / \varepsilon, \quad i=1, \ldots, n, n \in \mathbb{N} .
$$

Let $Y_{n}(t)$ be the number of $i \in\left\{1, \ldots, 2^{2 n}\right\}$ such that $F_{t}(i, n)$ occurs. We want to show that $P_{t}\left\{Y_{n}(t)>0\right\}$ is bounded below by a positive constant not depending on $n, \varepsilon$ or $t$. Observe using (4) that

$$
E_{t}\left(Y_{n}(t)\right)=\sum_{i=1}^{2^{2 n}} P_{t}\left(F_{t}(i, n)\right) \geq 2^{2 n} c 2^{-n} / \varepsilon=c 2^{n} / \varepsilon .
$$

Next, we show that there is a universal finite constant $K_{1}>0$ such that for $1 \leq$ $i<j \leq n$,

$$
P_{t}\left(F_{t}(i, n) \cap F_{t}(j, n)\right) \leq K_{1} 2^{-2 n} \varepsilon^{-1}\left|x_{j}-x_{i}\right|^{-\frac{1}{2}} .
$$

Indeed, notice that $F_{t}(i, n) \cap F_{t}(j, n)$ is contained in

$$
\begin{aligned}
F_{0} \cap & F_{1}(i, n) \cap F_{1}(j, n) \\
& \cap\left\{Y\left(\left(x_{i}+u\right) / V_{\varepsilon}, \tilde{\tau}_{\varepsilon}+\mu_{i} / t_{1}\right) \leq 2^{-n}, 0 \leq u \leq x_{j}-x_{i}\right\} \\
& \cap\left\{Y\left(\left(x_{j}+u\right) / V_{\varepsilon}, \tilde{\tau}_{\varepsilon}+\mu_{j} / t_{1}\right) \leq 2^{-n}, 0 \leq u \leq \varepsilon^{2}\right\} .
\end{aligned}
$$

Using again that (3) (with $i$ replaced by $j$ ) defines a Brownian motion conditionally independent of $\tilde{\mathcal{F}}_{x_{j} / V_{\varepsilon}}^{1}$, with speed in $[1,2]$ on $F_{1}(j, n)$, the conditional probability of the event in (8) given $\tilde{\mathcal{F}}_{x_{j} / V_{\varepsilon}}^{1}$ is $\leq K_{1} 2^{-n} / \varepsilon$ on $F_{1}(j, n)$. The event above becomes more likely if we remove $F_{1}(j, n)$ from the intersection. With the same reasoning, the conditional probability of the event in (7) given $\tilde{\mathcal{F}}_{x_{i} / V_{\varepsilon}}^{1}$ is $\leq K_{1} 2^{-n}\left|x_{j}-x_{i}\right|^{-\frac{1}{2}}$ on $F_{1}(i, n)$. This proves ([6). 
Notice that

$$
E_{t}\left(Y_{n}(t)^{2}\right)=\sum_{i=1}^{2^{2 n}} P_{t}\left(F_{t}(i, n)\right)+2 \sum_{i=1}^{2^{2 n}} \sum_{j=i+1}^{2^{2 n}} P_{t}\left(F_{t}(i, n) \cap F_{t}(j, n)\right) .
$$

One checks as in (6) - (8) that there is a universal finite constant $K_{2}>0$ such that $P_{t}\left(F_{t}(i, n)\right) \leq K_{2} 2^{-n} / \varepsilon$, so that this expression is not greater than

$$
\begin{aligned}
K_{2} \frac{2^{n}}{\varepsilon}+2 \sum_{i=1}^{2^{2 n}} \sum_{j=i+1}^{2^{2 n}} & K_{1}^{2} 2^{-2 n} \varepsilon^{-1}\left|(j-i) \varepsilon^{2} 2^{-2 n}\right|^{-\frac{1}{2}} \\
& =K_{2} \frac{2^{n}}{\varepsilon}+2 K_{1} 2^{-n} \varepsilon^{-2} \sum_{i=1}^{2^{2 n}} \sum_{j=i+1}^{2^{2 n}}|j-i|^{-\frac{1}{2}} .
\end{aligned}
$$

The double sum is bounded by

$$
\int_{0}^{2^{2 n}} d x \int_{x}^{2^{2 n}} d y|y-x|^{-\frac{1}{2}}=\frac{4}{3} 2^{3 n}
$$

Therefore,

$$
E_{t}\left(Y_{n}(t)^{2}\right) \leq K_{2} \frac{2^{n}}{\varepsilon}+\frac{8}{3} K_{1} \varepsilon^{-2} 2^{2 n} \leq K 2^{2 n} \varepsilon^{-2},
$$

for some universal constant $K$. From (5) and (9), we conclude that

$$
\begin{aligned}
E_{t}\left(Y_{n}(t)^{2}\right) & \leq K c^{-2} E_{t}\left(Y_{n}(t)\right)^{2} \\
& \leq K c^{-2} E_{t}\left(Y_{n}(t)^{2}\right) P_{t}\left\{Y_{n}(t)>0\right\},
\end{aligned}
$$

and so $P_{t}\left\{Y_{n}(t)>0\right\} \geq c^{2} / K>0$. Notice that this lower bound does not depend on $n, \varepsilon$ or $t$.

Set $G(t)=\lim _{\sup _{n \rightarrow \infty}}\left\{Y_{n}(t)>0\right\}$. Applying Fatou's Lemma, we conclude that

$$
P_{t}(G(t)) \geq \limsup _{n \rightarrow \infty} P_{t}\left\{Y_{n}(t)>0\right\} \geq c^{2} / K
$$

Notice that for $\omega \in G(t)$, there is a sequence $n_{k} \uparrow \infty$ such that $Y_{n_{k}}(t)(\omega)>0$, for all $k$, that is, there is $1 \leq i_{k} \leq n_{k}$ such that $\omega \in F_{t}\left(i_{k}, n_{k}\right)$. By passing to a subsequence, we can assume that $\left(x_{i_{k}}, \mu_{i_{k}}\right)$ converges to $(x, \mu)$, say. Then

$$
\begin{aligned}
\left(x / V_{\varepsilon}, \tilde{\tau}_{\varepsilon}+\mu / t_{1}\right) & \in\left[0, \varepsilon^{2} / V_{\varepsilon}\right] \times\left[\tilde{\tau}_{\varepsilon}, \tilde{\tau}_{\varepsilon}+\varepsilon^{2} / t_{1}\right], \\
Y\left(x / V_{\varepsilon}, \tilde{\tau}_{\varepsilon}+(\mu-v) / t_{1}\right) & =\lim _{k \rightarrow \infty} Y\left(x_{i_{k}} / V_{\varepsilon}, \tilde{\tau}_{\varepsilon}+\left(\mu_{i_{k}}-v\right) / t_{1}\right) \\
& \geq v^{\alpha},
\end{aligned}
$$

for $0<v<\mu$, and similarly,

$$
Y\left(\left(x_{i}+u\right) / V_{\varepsilon}, \tilde{\tau}_{\varepsilon}+\mu / t_{1}\right)<-u^{\alpha}, \quad \text { for } 0<u \leq \varepsilon^{2} .
$$

Finally, $Y\left(u / V_{\varepsilon}, \tilde{\tau}_{\varepsilon}\right)>\varepsilon / 2$ for $0 \leq u \leq \varepsilon^{2}$. So $G(t) \subset \tilde{G}_{\varepsilon}$, since $\left(u_{0}, v_{0}\right)=\left(x / V_{\varepsilon}, \tilde{\tau}_{\varepsilon}+\right.$ $\left.\mu / t_{1}\right)$ has the required properties. This proves (a).

(b) By Fatou's lemma and (a), we obtain $P_{t}(\tilde{F}) \geq c_{0}>0$. By Lemma 6 , we conclude that $P_{t}(\tilde{F})=1$. This proves Lemma 7 .

The next two lemmas establish some technical properties of the Brownian sheet. For $t \in \mathbb{R}_{+}^{2}$, set $\mathcal{F}_{t}=\sigma\{W(s), s \leq t\}$ and recall that a stopping point for $W$ is a random variable $T$ such that for each $t \in \mathbb{R}_{+}^{2},\{T \leq t\} \in \mathcal{F}_{t}$. 
Lemma 8. Set $T=\left(T_{1}, 1\right)$, where

$$
T_{1}=\inf \left\{t_{1}>1: W\left(t_{1}, 1\right)=0\right\} .
$$

Then $T$ is a stopping point and, for $t_{1}>1$, the conditional distribution of $\left(W^{T}(u, v)\right.$ $\left.=W\left(T_{1}+u, 1+v\right),(u, v) \in \mathbb{R}_{+}^{2}\right)$ given $T=\left(t_{1}, 1\right)$ is equal to the distribution of $Y$ under $P_{\left(t_{1}, 1\right)}$.

Proof. The fact that $T$ is a stopping point follows immediately from the definitions. Set

$$
\begin{aligned}
& B_{1}(u)=W\left(T_{1}+u, 1\right), \quad B_{2}(v)=W\left(T_{1}, 1+v\right), \\
& \hat{W}(u, 0)=W\left(T_{1}+u, 1+v\right)-W\left(T_{1}+u, 1\right)-W\left(T_{1}, 1+v\right) .
\end{aligned}
$$

Because $W(T)=0$ a.s., $\hat{W}$ is a Brownian sheet that is independent of $\mathcal{F}_{T}$ (see e.g. 11, Theorem 1.6]), and given $T=\left(t_{1}, 1\right), B_{1}$ and $B_{2}$ are conditionnally independent Brownian motions, independent of $\hat{W}$, with speeds 1 and $\sqrt{t_{1}}$, respectively. Because

$$
W^{T}(u, v)=B_{1}(u)+B_{2}(v)+\hat{W}(u, v),
$$

the conditional distribution of $W^{T}$ given $T=\left(t_{1}, 1\right)$ is equal to the distribution of $Y$ under $P_{\left(t_{1}, 1\right)}$.

The following lemma gives bounds on certain hitting times of the Brownian sheet restricted to certain lines. These bounds are rather crude, but are sufficient for our purposes.

Lemma 9. Fix $\beta \in] 0,1]$. With $T$ and $T_{1}$ as in Lemma 8, define

$$
\begin{aligned}
\tau_{\varepsilon} & =\inf \left\{v>0: W\left(T_{1}, 1+v\right)=\varepsilon\right\}, \\
\tau_{\varepsilon}^{\prime} & =\inf \left\{v>0: W\left(T_{1}, 1+v\right)=-\varepsilon^{1-\beta}\right\}, \\
\sigma_{\varepsilon} & =\inf \left\{u>0:\left|W\left(T_{1}-u, 1\right)\right|=\varepsilon\right\} .
\end{aligned}
$$

(a) With probability 1 , there is $\varepsilon_{0}>0$ such that for all $0<\varepsilon<\varepsilon_{0}, \sigma_{\varepsilon} \leq \varepsilon^{2(1-\beta)}$.

(b) With probability 1 , there is $\varepsilon_{0}>0$ such that for all $0<\varepsilon<\varepsilon_{0}, \tau_{\varepsilon} \leq \varepsilon^{2-\beta}$ and $\tau_{\varepsilon} \leq \tau_{\varepsilon}^{\prime}$.

Proof. (a) Consider the event

$$
A(n)=\left\{\sigma_{2^{-n}}>\frac{1}{4}\left(2^{-n}\right)^{2(1-\beta)}\right\}
$$

(the factor $1 / 4$ will be used below, to get a statement valid for all $\varepsilon>0$ ). Set

$$
b(u)=\left|W\left(T_{1}-u, 1\right)\right|
$$

and recall [2, Lemma 2.4] that $\left(b(u), 0 \leq u \leq T_{1}-1\right)$ is a Bessel(3) process run until it last hits an $N(0,1)$-Gaussian random variable. Set $b_{*}(u)=\sup _{0 \leq v \leq u} b(v)$. By the scaling property of this process,

$$
\begin{aligned}
P(A(n)) & \leq P\left\{b_{*}\left(\frac{1}{4}\left(2^{-n}\right)^{2(1-\beta)}\right)<2^{-n}\right\} \\
& =P\left\{b_{*}(1) \leq 2^{1-n \beta}\right\} .
\end{aligned}
$$

Because $b$ has the same law as the modulus of a 3-dimensional Brownian motion, for large $n$, this is (much) smaller than $\left(2^{1-n \beta}\right)^{3}$. Therefore, $\sum_{n=1}^{\infty} P(A(n))<\infty$, and from the Borel-Cantelli lemma, we conclude that $P\left(\lim \sup _{n \rightarrow \infty} A(n)\right)=0$. 
Fix $\omega \notin \limsup _{n \rightarrow \infty} A(n)$. There is $n_{0}(\omega)>0$ such that $\sigma_{2^{-n}}(\omega) \leq \frac{1}{4}\left(2^{-n}\right)^{2(1-\beta)}$ for $n \geq n_{0}(\omega)$. Set $\varepsilon_{0}=2^{-n_{0}(\omega)}$ and consider $0<\varepsilon<\varepsilon_{0}(\omega)$. There is $n \geq n_{0}(\omega)$ such that $2^{-(n+1)}<\varepsilon \leq 2^{-n}$. Therefore,

$$
\sigma_{\varepsilon}(\omega)<\sigma_{2^{-n}}(\omega) \leq \frac{1}{4}\left(2^{-n}\right)^{2(1-\beta)} \leq \frac{1}{4}(2 \varepsilon)^{2(1-\beta)} \leq \varepsilon^{2(1-\beta)} .
$$

This proves $(\mathrm{a})$.

(b) This follows the same idea as in (a). Consider the event

$$
C(n)=\left\{\tau_{2^{-n}}>\frac{1}{4 T_{1}}\left(2^{-n}\right)^{2-\beta}\right\} \cup\left\{\tau_{2^{-n}}^{\prime}<\frac{1}{T_{1}}\left(2^{-n}\right)^{2-\beta}\right\} .
$$

Let $B^{*}(u)=\sup _{0 \leq v \leq u} B(u)$, where $B(u)$ is a standard Brownian motion independent of $W$. Because $v \mapsto W\left(T_{1}, 1+v\right)$ has the same law as $v \mapsto \sqrt{T_{1}} B(v)$, the scaling property of Brownian motion implies that

$$
\begin{aligned}
P(C(n)) & \leq P\left\{B^{*}(1) \leq 2^{1-n \beta / 2}\right\}+P\left\{B^{*}(1) \geq 2^{n \beta / 2}\right\} \\
& \leq C 2^{-n \beta / 2} .
\end{aligned}
$$

Therefore $\sum_{n=1}^{\infty} P(C(n))<\infty$ and $P\left(\lim \sup _{n \rightarrow \infty} C(n)\right)=0$.

Fix $\omega \notin \lim \sup _{n \rightarrow \infty} C(n)$. There is $n_{0}(\omega)>0$ such that for $n \geq n_{0}(\omega)$,

$$
\tau_{2^{-n}}(\omega) \leq \frac{1}{4 T_{1}(\omega)}\left(2^{-n}\right)^{2-\beta} \text { and } \tau_{2^{-n}}^{\prime}(w) \geq \frac{1}{T_{1}(\omega)}\left(2^{-n}\right)^{2-\beta} \text {. }
$$

Because $T_{1}(\omega) \geq 1$, we conclude, as in part (a) above, that $\tau_{\varepsilon}(\omega) \leq \varepsilon^{2-\beta}$ for $0<\varepsilon<\varepsilon_{0}(\omega)=2^{-n_{0}(\omega)}$. Further, for such an $\varepsilon$, there is $n \geq n_{0}(\omega)$ such that $2^{-(n+1)}<\varepsilon \leq 2^{-n}$. Then

$$
\begin{aligned}
\tau_{\varepsilon}(\omega) & \leq \tau_{2^{-n}}(\omega) \leq \frac{1}{4 T_{1}(\omega)}\left(2^{-n}\right)^{2-\beta} \leq \frac{1}{T_{1}(\omega)}\left(2^{-(n+1)}\right)^{2-\beta} \\
& \leq \tau_{2^{-(n+1)}}^{\prime}(\omega) \leq \tau_{\varepsilon}^{\prime}(\omega) .
\end{aligned}
$$

This proves $(b)$.

Lemma 10. Let $T_{1}$ be as in Lemma 8 and let $\tau_{\varepsilon}$ be as in Lemma 9. A.s. on $\{W(1,1)>0\}$, there is $\varepsilon_{0}>0$ such that for $0<\varepsilon<\varepsilon_{0},\left(T_{1}, 1+\tau_{\varepsilon}\right) \in C(1,1)$, where $C(1,1)$ denotes the component of $\left\{\left(t_{1}, t_{2}\right) \in \mathbb{R}_{+}^{2}: W\left(t_{1}, t_{2}\right)>0\right\}$ that contains $(1,1)$.

Proof. Set $\beta=1 / 10$. By Lemma 9, a.s., there is $\varepsilon_{0}>0$ such that for $0<\varepsilon<\varepsilon_{0}$,

$$
\tau_{\varepsilon} \leq \min \left(\tau_{\varepsilon}^{\prime}, \varepsilon^{2-\beta}\right) \quad \text { and } \quad \sigma_{2 \varepsilon^{1-\beta}} \leq\left(2 \varepsilon^{1-\beta}\right)^{2(1-\beta)} .
$$

Let $\Gamma_{1}$ (resp. $\Gamma_{2}$ ) be the vertical (resp. horizontal) segment defined by

$$
\begin{aligned}
& \Gamma_{1}=\left\{T_{1}-\sigma_{2 \varepsilon^{1-\beta}}\right\} \times\left[1,1+\tau_{\varepsilon}\right], \\
& \Gamma_{2}=\left[T_{1}-\sigma_{2 \varepsilon^{1-\beta}}, T_{1}\right] \times\left\{1+\tau_{\varepsilon}\right\},
\end{aligned}
$$

and set $\Gamma=\Gamma_{1} \cup \Gamma_{2}$. Clearly, $\left(T_{1}-\sigma_{2 \varepsilon^{1-\beta}}, 1\right) \in C(1,1)$, and $W\left(T_{1}, T_{1}+\tau_{\varepsilon}\right)=\varepsilon>0$. It therefore suffices to show that $W>0$ along $\Gamma$.

Let $B_{2}(u)$ be defined as in (10), $b(u)$ as in (11), and set

$$
x(u, v)=W\left(T_{1}, 1+v\right)-W\left(T_{1}-u, 1+v\right)+W\left(T_{1}-u, 1\right) .
$$

Because $T_{1} \geq 1$ and $W(T)=0,\left(x(u, v),(u, v) \in[0,1] \times \mathbb{R}_{+}\right)$is a Brownian sheet and

$$
W\left(T_{1}-u, 1+v\right)=b(u)+B_{2}(v)-x(u, v) .
$$


An upper bound on $x$ is given by the law of the iterated logarithm for the Brownian sheet [7, Theorem 2.2]:

$$
0<a<\frac{1}{2} \Rightarrow \lim _{\varepsilon \downarrow 0} \sup _{0<h \leq \varepsilon, 0<k<\varepsilon}(h k)^{-a}|x(h, k)|=0 .
$$

So, after possibly reducing the value of $\varepsilon_{0}$, we can assume that for $0<\varepsilon<\varepsilon_{0}$,

$$
\sup _{0<h<\varepsilon, 0<k<\varepsilon}(h k)^{-\frac{2}{3}}|x(h, k)| \leq 1,
$$

and, in particular, recalling that $\beta=1 / 10$,

(12) $|x(h, k)| \leq\left(\left(2 \varepsilon^{1-\beta}\right)^{2(1-\beta)} \cdot \varepsilon^{2-\beta}\right)^{2 / 3} \leq 4 \varepsilon^{2} \quad$ for $(h, k) \in\left[0, \sigma_{2 \varepsilon^{1-\beta}}\right] \times\left[0, \tau_{\varepsilon}\right]$.

Let $v$ be such that $\left(T_{1}-\sigma_{2 \varepsilon^{1-\beta}}, 1+v\right) \in \Gamma_{1}$. Then, because $\tau_{\varepsilon} \leq \tau_{\varepsilon}^{\prime}$ and by (12),

$$
\begin{aligned}
W\left(T_{1}-\sigma_{2 \varepsilon^{1-\beta}}, 1+v\right) & =2 \varepsilon^{1-\beta}+B_{2}(v)-x\left(\sigma_{2 \varepsilon^{1-\beta}}, v\right) \\
& \geq 2 \varepsilon^{1-\beta}-\varepsilon^{1-\beta}-4 \varepsilon^{2} \\
& >0 .
\end{aligned}
$$

Let $u$ be such that $\left(T_{1}-u, 1+\tau_{\varepsilon}\right) \in \Gamma_{2}$. Then, because $b(u)>0$ and by (12),

$$
\begin{aligned}
W\left(T_{1}-u, 1+\tau_{\varepsilon}\right) & =b(u)+\varepsilon-x\left(u, \tau_{\varepsilon}\right) \\
& \geq 0+\varepsilon-4 \varepsilon^{2} \\
& >0 .
\end{aligned}
$$

This establishes that $W>0$ along $\Gamma$ and proves the lemma.

The next lemma is the analogue at level 0 of the corresponding result of [2] Theorem 2.1] for level 1, which we state without proof.

Lemma 11. Let $T$ be as in Lemma 8. With probability one, there exists a sequence of disjoint contours $\left(\Gamma_{n}, n \geq 1\right)$ with diameter tending to 0 as $n \rightarrow \infty$, each of which contains $T$ in its interior and on which $W$ has the same sign as $W(1,1)$.

We now have all the necessary ingredients needed to prove Theorem 2

Proof of Theorem 2. It suffices to show that a.s. on $\{W(1,1)>0\}, C(1,1)$ is hinged to infinitely many negative bubbles.

Fix $\beta=1 / 10$. Let $T, T_{1}$ and $\tau_{\varepsilon}$ be as in Lemmas 8 and 9 , and let $S^{\varepsilon}=\left(S_{1}^{\varepsilon}, S_{2}^{\varepsilon}\right)$, where $S_{1}^{\varepsilon}=T_{1}$ and $S_{2}^{\varepsilon}=1+\tau_{\varepsilon}$. Fix $\alpha>\frac{1}{2}$. Let $G_{\varepsilon}$ be the event "there exists $\left(s_{1}, s_{2}\right) \in\left[S_{1}^{\varepsilon}, S_{1}^{\varepsilon}+\varepsilon^{2}\right] \times\left[S_{2}^{\varepsilon}, S_{2}^{\varepsilon}+\varepsilon^{2}\right]$ such that $W>0$ on $\left(\left[S_{1}^{\varepsilon}, s_{1}\right] \times\left\{S_{2}^{\varepsilon}\right\}\right) \cup\left(\left\{s_{1}\right\} \times\right.$ $\left[S_{2}^{\varepsilon}, s_{2}[)\right.$ and there is $\delta_{1}>0$ such that $W\left(s_{1}, s_{2}-u\right)>u^{\alpha}$ and $W\left(s_{1}+u, s_{2}\right)<-u^{\alpha}$ for $0<u<\delta_{1}$."

Because $W(T)=0$ a.s., the event $G_{\varepsilon}$ is a statement about the behavior of $W^{T}$ (defined in Lemma 8). Formally, let $\tilde{F}$ and $\tilde{G}_{\varepsilon}$ be the event defined in (and just before) (2), and let $D_{\varepsilon} \in \tilde{\mathcal{B}}$ be such that

$$
\tilde{G}_{\varepsilon}=\left\{Y \in D_{\varepsilon}\right\} .
$$

Then, comparing definitions, we clearly have $G_{\varepsilon}=\left\{W^{T} \in D_{\varepsilon}\right\}$.

Let $F=\limsup _{n \rightarrow \infty} G_{1 / n}$ and $D=\limsup _{n \rightarrow \infty} D_{1 / n}$, so that $F=\left\{W^{T} \in D\right\}$. For $t \in \mathbb{R}_{+}^{2}$, let $P_{t}$ be the probability measure on $(\tilde{\Omega}, \tilde{\mathcal{B}})$ defined before Lemma $[6$ 
Using the fact that the conditional distribution of $W^{T}$ given $T$ is a.s. equal to the distribution of $Y$ under $P_{T}$, we see that

$$
\begin{aligned}
P(F) & =E(P(F \mid T))=E\left(P\left\{W^{T} \in D \mid T\right\}\right) \\
& =E\left(P_{T}\{Y \in D\}\right) \\
& =E\left(P_{T}(\tilde{F})\right) \\
& =1
\end{aligned}
$$

by Lemma 7(b). It follows that a.s., $G_{1 / n}$ occurs for infinitely many $n$. Therefore, by Lemma 10, for a.a. $\omega \in\{W(1,1)>0\}$ and large enough $n$ such that $\omega \in G_{1 / n}$, $C(1,1)$ is hinged to a negative bubble at some point within $\left[T_{1}, T_{1}+\varepsilon^{2}\right] \times\left[1,1+2 \varepsilon^{1.9}\right]$, with a growth exponent $\leq \alpha$ at the corner of the bubble. By Lemma 11, infinitely many of these negative bubbles are distinct. This proves that on $\{W(1,1)>0\}$, $C(1,1)$ is hinged to infinitely many negative bubbles with growth exponent at the corner of the hinge $\leq \alpha$ (recall that $\alpha>\frac{1}{2}$ was fixed before (2) ). To check that the distance exponent of these hinges is $\leq 2 \alpha /(1-4 \varepsilon)$, for all $\varepsilon>0$, we shall use the Lévy modulus of continuity of the Brownian sheet [11, Proposition 1.4]: for $\varepsilon>0$ fixed,

$$
|W(t)-W(s)| \leq K|s-t|^{\frac{1}{2}-\varepsilon}
$$

where $K$ is an a.s. finite random variable. Let $\left(t_{1}, t_{2}\right)$ be the corner of a hinge with growth exponent $\leq \alpha$. For small $v>0, W\left(t_{1}, t_{2}-v\right)>v^{\alpha}$. Therefore, for $s$ such that $\left|s-\left(t_{1}, t_{2}-v\right)\right| \leq v^{2 \alpha /(1-4 \varepsilon)}$,

$$
\begin{aligned}
W(s) & \geq W\left(t_{1}, t_{2}-v\right)-K\left|s-\left(t_{1}, t_{2}-v\right)\right|^{\frac{1}{2}-\varepsilon} \\
& \geq v^{\alpha}-K v^{\alpha(1-2 \varepsilon) /(1-4 \varepsilon)} \\
& >0
\end{aligned}
$$

for small $v>0$; so $d\left(\left(t_{1}, t_{2}-v\right), L(0)\right)>v^{2 \alpha /(1-4 \varepsilon)}$. This concludes the proof of Theorem 2

\section{Proof of Theorem 4}

Recall that we have set

$$
X\left(s_{1}, s_{2}\right)=B_{1}\left(s_{1}\right)-B_{2}\left(s_{2}\right),
$$

where $B_{1}, B_{2}$ are two independent two-sided Brownian motions. For definiteness, we treat the case where $B_{1}(0)=B_{2}(0)=0$, but the only consequence of this assumption that we will use is that $X\left(s_{1}, s_{2}\right) \neq 0$ a.s., for $\left(s_{1}, s_{2}\right) \neq(0,0)$. The process $X$ is indexed by $\mathbb{R}^{2}$, but the proofs carry over to the case where the index set is the first quadrant.

We will term a cross a pair $C$ of line segments

$$
\{] s_{1}, t_{1}[\times\{v\},\{u\} \times] s_{2}, t_{2}[\}
$$

such that $u \in] s_{1}, t_{1}[$ and $v \in] s_{2}, t_{2}[$ (that is, the two open line segments intersect). The point $(u, v)$ is the center of the cross. Given a cross $C$ as above, we set $R(C)=\left[s_{1}, t_{1}\right] \times\left[s_{2}, t_{2}\right]$, and call $R(C)$ the rectangle generated by $C$.

A complete description of bubbles for the process $X$ is given in [3, Section 2]: see, in particular, Remark 2.3(b) of that paper. We briefly recall the properties of these bubbles that we will need further on. 
Every bubble is bounded, and to each bubble $E$, there corresponds a (random) distinguished cross

$$
C(E)=\{] \sigma_{1}, \tau_{1}\left[\times\left\{S_{2}\right\},\left\{S_{1}\right\} \times\right] \sigma_{2}, \tau_{2}[\}
$$

such that $C(E) \subset E \subset R(C(E)$ ), with the following additional properties.

If the bubble $E$ is positive, then the interval $] \sigma_{1}, \tau_{1}[$ is an excursion interval for $B_{1}$ from some (random) value $m$ up to some (random) maximum value $M>m$, and $B_{1}\left(S_{1}\right)=M, B_{1}\left(\sigma_{1}\right)=B_{1}\left(\tau_{1}\right)=m$, while the interval $] \sigma_{2}, \tau_{2}$ [ is an excursion interval for $B_{2}$ from value $M$ down to the minimum value $m$, and $B_{2}\left(S_{2}\right)=m$, $B_{2}\left(\sigma_{2}\right)=B_{2}\left(\tau_{2}\right)=M$. The positive value $M-m$ at the point $\left(S_{1}, S_{2}\right)$ is the unique maximum of $X$ on $R(C(E))$.

If the bubble $E$ is negative, then the interval $] \sigma_{2}, \tau_{2}[$ is an excursion interval for $B_{2}$ from some (random) value $m$ up to some (random) maximum value $M>m$, and $B_{2}\left(S_{2}\right)=M, B_{2}\left(\sigma_{2}\right)=B_{2}\left(\tau_{2}\right)=m$, while the interval $] \sigma_{1}, \tau_{1}$ [ is an excursion interval for $B_{1}$ from value $M$ down to the minimum value $m$, and $B_{1}\left(S_{1}\right)=m$, $B_{1}\left(\sigma_{1}\right)=B_{1}\left(\tau_{1}\right)=M$. The negative value $m-M$ at the point $\left(S_{1}, S_{2}\right)$ is the unique minimum of $X$ on $R(C(E))$.

The distinguished cross is the only cross contained in the bubble $E$ such that

$$
X\left(S_{1}, \sigma_{2}\right)=X\left(S_{1}, \tau_{2}\right)=X\left(\sigma_{1}, S_{2}\right)=X\left(\tau_{1}, S_{2}\right)=0,
$$

$X \neq 0$ on this cross, and, if the bubble is positive (resp. negative), the maximum (resp. minimum) of $X$ over the segment $\left[\sigma_{1}, \tau_{1}\right] \times\left\{S_{2}\right\}$ is equal to its maximum (resp. minimum) over the segment $\left\{S_{1}\right\} \times\left[\sigma_{2}, \tau_{2}\right]$, and this maximum (resp. minimum) is attained at the center $\left(S_{1}, S_{2}\right)$ of the cross.

Lemma 12. Given a bubble $E$ with distinguished cross $C(E)$ as described above, the four corners of $R(C(E))$ are a.s. in a single bubble and

$$
\begin{aligned}
X\left(\sigma_{1}, \sigma_{2}\right) & =X\left(\tau_{1}, \sigma_{2}\right)=X\left(\sigma_{1}, \tau_{2}\right)=X\left(\tau_{1}, \tau_{2}\right) \\
& =-X\left(S_{1}, S_{2}\right) .
\end{aligned}
$$

Proof. It is noted in [3] that the four corners of $R(C(E))$ are a.s. in a single bubble: see the proof of Lemma 5.3 of that paper.

For a rectangle $R=\left[s_{1}, t_{1}\right] \times\left[s_{2}, t_{2}\right]$, set

$$
\Delta_{R} X=X\left(t_{1}, t_{2}\right)-X\left(s_{1}, t_{2}\right)-X\left(t_{1}, s_{2}\right)+X\left(s_{1}, s_{2}\right),
$$

and notice from (13) that $\Delta_{R} X=0$, for any rectangle $R$. Therefore,

$$
X\left(S_{1}, S_{2}\right)-X\left(S_{1}, \sigma_{2}\right)-X\left(\sigma_{1}, S_{2}\right)+X\left(\sigma_{1}, \sigma_{2}\right)=0 .
$$

Because $X\left(S_{1}, \sigma_{2}\right)=X\left(\sigma_{1}, S_{2}\right)=0$ by (14), it follows that $X\left(\sigma_{1}, \sigma_{2}\right)=-X\left(S_{1}, S_{2}\right)$. A similar argument proves the statement for the other three corners of $R(C(E))$.

We now examine measurability properties of the variables $S_{i}, \tau_{i}$ and $\sigma_{i}$.

Lemma 13. Given $r \in \mathbb{R}^{2} \backslash\{(0,0)\}$, let $E^{r}$ denote the bubble that contains $r$, and let $S_{i}, \tau_{i}$ and $\sigma_{i}$ be the random variables defined above for the bubble $E=E^{r}$. Then $S_{i}, \tau_{i}$ and $\sigma_{i}$ are measurable with respect to $\mathcal{G}^{\prime}$.

Proof. For $t \in \mathbb{R}^{2}$, set $D(t)=D_{1}(t)+D_{2}(t)+D_{3}(t)+D_{4}(t)$, where

$$
\begin{array}{ll}
D_{1}(t)=\inf \{u: X(t+(u, 0))=0\}, & D_{3}(t)=\inf \{u: X(t+(-u, 0))=0\}, \\
D_{2}(t)=\inf \{u: X(t+(0, u))=0\}, & D_{4}(t)=\inf \{u: X(t+(0,-u))=0\} .
\end{array}
$$


Clearly, $D(t)$ is measurable with respect to $\mathcal{G}^{\prime}$, and by path continuity of $X$, a.s., $t \mapsto D(t)$ is lower semicontinuous. Let $A^{\prime}(s, t)$ be the event denoted $A(s, t)$ in the proof of Theorem [3, but with $W$ replaced by $X$. Then $A^{\prime}(s, t) \in \mathcal{G}^{\prime}$, and so for $r \in \mathbb{R}^{2} \backslash\{(0,0)\}$ fixed,

$$
D^{\prime}(r) \stackrel{\text { def }}{=} \sup _{t \in \mathbb{R}^{2}}\left(D(t) 1_{A^{\prime}(r, t)}\right)
$$

is $\mathcal{G}^{\prime}$-measurable. The properties of bubbles mentioned above imply that $D^{\prime}(r)<\infty$ a.s., and $D\left(S_{1}, S_{2}\right)=D^{\prime}(r)$. By lower semicontinuity of $t \mapsto D(t)$, there exists a sequence of rational points $q_{n} \in E^{r}$ such that $D\left(q_{n}\right) 1_{A\left(r, q_{n}\right)} \rightarrow D^{\prime}(r)$ as $n \rightarrow \infty$. The properties of bubbles of $X$ discussed above imply that for any sequence $\left(q_{n}\right)$ such that $D\left(q_{n}\right) 1_{A\left(r, q_{n}\right)} \rightarrow D^{\prime}(r)$, it must be the case that $q_{n} \rightarrow\left(S_{1}, S_{2}\right)$.

Let $\left(q^{(n)}, n \in \mathbb{N}\right)$ be an enumeration of $\mathbb{Q}^{2}$. For $m \geq 1$, let $\tau_{m}=\inf \{n$ : $\left.D\left(q^{(n)}\right) 1_{A\left(r, q^{(n)}\right)}>D^{\prime}(r)-1 / m\right\}$. Then $D\left(q^{\left(\tau_{m}\right)}\right) 1_{A\left(r, q^{\left(\tau_{m}\right)}\right)} \rightarrow D^{\prime}(r)$, and so $q^{\left(\tau_{m}\right)} \rightarrow\left(S_{1}, S_{2}\right)$. Therefore, $S_{i}$ is measurable with respect to $\mathcal{G}^{\prime}, i=1,2$. The measurability of $\tau_{i}$ and $\sigma_{i}$ with respect to $\mathcal{G}^{\prime}$ is now immediate.

Given $r=\left(r_{1}, r_{2}\right) \neq(0,0)$ (so that a.s., $r$ is within a bubble), let $E^{r}=E_{1}^{r}$ denote the bubble that contains $r$. We use the term the cross sequence of $r$ for the sequence of crosses

$$
\left(C_{i}^{r}=\{] \sigma_{1}^{i}(r), \tau_{1}^{i}(r)\left[\times\left\{S_{2}^{i}(r)\right\},\left\{S_{1}^{i}(r)\right\} \times\right] \sigma_{2}^{i}(r), \tau_{2}^{i}(r)[\}, i \geq 1\right),
$$

such that $C_{1}^{r}=C\left(E_{1}^{r}\right)$, and for $i \geq 1, C_{i+1}^{r}$ is the distinguished cross for the bubble that contains the four corner vertices of $R\left(C_{i}^{r}\right)$. The bubble corresponding to $C_{i}^{r}$ will be denoted by $E_{i}^{r}$. The next two lemmas are direct consequences of Lemmas 13 and 12 .

Lemma 14. The sequence $\left(C_{i}^{r}, i=1,2, \ldots\right)$ is measurable with respect to $\mathcal{G}^{\prime}$.

Lemma 15. For $i \geq 1$, the sign of $E_{i}^{r}$ is the opposite of the sign of $E_{i+1}^{r}$.

Proof. Because $\left(\sigma_{1}^{i}(r), \sigma_{2}^{i}(r)\right) \in E_{i+1}^{r}$ and $\left(S_{1}^{i}(r), S_{2}^{i}(r)\right) \in E_{i}^{r}$, the conclusion follows immediately from Lemma 12 .

We now show that the union over $i \geq 1$ of the rectangles $R\left(C_{i}^{r}\right)$ is $\mathbb{R}^{2}$.

Lemma 16. With probability one, for all $r \neq(0,0)$ and $N>0$, there exists $n_{0}=n_{0}(N, r)<\infty$ such that

$$
[-N, N]^{2} \subset R\left(C_{n_{0}}^{r}\right) .
$$

Proof. The minima and maxima of $B_{1}$ over $] \sigma_{1}^{i}(r), \tau_{1}^{i}(r)\left[\right.$ and also of $B_{2}$ over ] $\sigma_{2}^{i}(r), \tau_{2}^{i}(r)$ [ will be denoted $m_{i}(r)$ and $M_{i}(r)$. We note that for $j=1,2$,

$$
\sigma_{j}^{i+1}(r)<\sigma_{j}^{i}(r)<\tau_{j}^{i}(r)<\tau_{j}^{i+1}(r)
$$

and

$$
m_{i+1}(r)<m_{i}(r)<M_{i}(r)<M_{i+1}(r),
$$

since otherwise the independent Brownian motions $B_{1}$ and $B_{2}$ would have shared values at local extrema.

Every bubble contains a point with rational coordinates. So in order to prove the lemma, it suffices to show that for every fixed $r \neq(0,0)$, a.s.,

$$
\lim _{i \rightarrow \infty} \sigma_{1}^{i}(r)=\lim _{i \rightarrow \infty} \sigma_{2}^{i}(r)=-\infty \quad \text { and } \quad \lim _{i \rightarrow \infty} \tau_{1}^{i}(r)=\lim _{i \rightarrow \infty} \tau_{2}^{i}(r)=+\infty .
$$


Without loss of generality, we assume that $X$ is positive on $E_{1}^{r}$. In this case, by Lemma [15, for odd $i \geq 1, X$ is positive on $E_{i}^{r}$, therefore

$$
\begin{aligned}
& m_{i}(r)=B_{1}\left(\sigma_{1}^{i}(r)\right)=B_{1}\left(\tau_{1}^{i}(r)\right)=B_{2}\left(S_{2}^{i}(r)\right), \\
& M_{i}(r)=B_{2}\left(\sigma_{2}^{i}(r)\right)=B_{2}\left(\tau_{2}^{i}(r)\right)=B_{1}\left(S_{1}^{i}(r)\right),
\end{aligned}
$$

and $X$ is negative on $E_{i+1}^{r}$, therefore

$$
\begin{aligned}
& m_{i+1}(r)=B_{2}\left(\sigma_{2}^{i+1}(r)\right)=B_{2}\left(\tau_{2}^{i+1}(r)\right)=B_{1}\left(S_{1}^{i+1}(r)\right), \\
& M_{i+1}(r)=B_{1}\left(\sigma_{1}^{i+1}(r)\right)=B_{1}\left(\tau_{1}^{i+1}(r)\right)=B_{2}\left(S_{2}^{i+1}(r)\right) .
\end{aligned}
$$

In particular,

$$
\begin{aligned}
B_{1}\left(\sigma_{1}^{i+1}(r)\right)-B_{1}\left(\sigma_{1}^{i}(r)\right) & =M_{i+1}(r)-m_{i}(r) \\
\geq & M_{1}(r)-m_{1}(r) \\
& >0
\end{aligned}
$$

and

$$
\begin{aligned}
B_{1}\left(\sigma_{1}^{i+1}(r)\right)-B_{1}\left(\sigma_{1}^{i+2}(r)\right) & =M_{i+1}(r)-m_{i+2}(r) \\
& \geq M_{1}(r)-m_{1}(r) \\
& >0 .
\end{aligned}
$$

Therefore, for all $i \geq 1$,

$$
\left|B_{1}\left(\sigma_{1}^{i}(r)\right)-B_{1}\left(\sigma_{1}^{i+1}(r)\right)\right| \geq M_{1}(r)-m_{1}(r)>0 .
$$

Because $B_{1}(\cdot)$ is continuous, this implies, together with (16), that $\lim _{i \rightarrow \infty} \sigma_{1}^{i}(r)=$ $-\infty$. One obtains the other three limits in (17) in a similar way.

Lemma 17. For any two fixed $r, r^{\prime} \in \mathbb{R}^{2} \backslash\{(0,0)\}$, there exist (random) a.s. nonnegative integers $N$ and $M$ such that $E_{N}^{r}=E_{M}^{r^{\prime}}\left(\right.$ and $R\left(C_{N}^{r}\right)=R\left(C_{M}^{r}\right)$ ) a.s. In fact, one can take

$$
\begin{aligned}
N & =\inf \left\{n>1: r^{\prime} \in R\left(C_{n-1}^{r}\right)\right\}, \\
M & =\inf \left\{m \geq 1: E_{m}^{r^{\prime}} \cap R\left(C_{N-1}^{r}\right)^{c} \neq \emptyset\right\} .
\end{aligned}
$$

With this choice, $N$ and $M$ are $\mathcal{G}^{\prime}$-measurable.

Proof. Let $N$ and $M$ be defined as in the statement of the lemma: these random variables are $\mathcal{G}^{\prime}$-measurable by Lemma 14. Note that by Lemma [16, $N<\infty$ and $M<\infty$ a.s. Observe that $E_{M}^{r^{\prime}}$ contains points in $R\left(C_{M-1}^{r^{\prime}}\right)$, and $R\left(C_{M-1}^{r^{\prime}}\right)$ is contained in $R\left(C_{N-1}^{r}\right)$. So by the definition of a bubble, there must exist a path in $E_{M}^{r^{\prime}}$ from inside $R\left(C_{N-1}^{r}\right)$ to strictly outside $R\left(C_{N-1}^{r}\right)$ on which $X$ is nonzero. This path crosses the boundary of $R\left(C_{N-1}^{r}\right)$, at a point where $X$ is nonzero, which therefore belongs to $E_{N}^{r}$. It follows that $E_{N}^{r}=E_{M}^{r^{\prime}}$.

Proof of Theorem 4. It suffices to show that for $r \in \mathbb{R}^{2} \backslash\{(0,0)\}$, sign $X(r)$ is measurable with respect to $\sigma\{\operatorname{sign} X(1,1)\} \vee \mathcal{G}^{\prime}$. We apply Lemma 17 to points $r$ and $r^{\prime}=(1,1)$. As noted in Lemma 14, given $\mathcal{G}^{\prime}$, we can determine the sequences $E_{i}^{r}$ and $E_{i}^{r^{\prime}}, i \geq 1$. By Lemma 17, there exist $\mathcal{G}^{\prime}$-measurable random variables $N$ and $M$ such that $E_{N}^{r}=E_{M}^{r^{\prime}}$. It follows from the alternating sign property of Lemma 15] that $X(r) X\left(r^{\prime}\right)(-1)^{N+M}>0$ a.s. Thus $\{X(r)>0\}=\left\{X(1,1)(-1)^{N+M}>0\right\}$, and this event clearly belongs to $\sigma(\operatorname{sign} X(1,1)) \vee \mathcal{G}^{\prime}$. Theorem 4 is proved. 


\section{Appendix, By John B. Walsh}

The following result concerning non-independence of excursions of the Brownian sheet was inspired by the results in Theorems 2 and 4 .

Theorem 18. $\sigma\left\{|W(s)|, s \in \mathbb{R}_{+}^{2}\right\} \vee \sigma\{\operatorname{sign} W(1,1)\}=\sigma\left\{W(s), s \in \mathbb{R}_{+}^{2}\right\}$. In other words, given the absolute value of the sheet in the non-negative quadrant and its sign at a single point, one can determine the sign of the sheet everywhere in the quadrant.

The proof of this theorem is based on the following lemma for Brownian motions.

Lemma 19. Fix $a>0$ and $b>0$. Let $B_{1}$ and $B_{2}$ be independent Brownian motions. For $u \geq 0$, set $X_{1}(u)=a B_{1}(u), X_{2}(u)=a B_{1}(u)+b B_{2}(u)$, and define the two $\sigma$-fields

$$
\begin{aligned}
& \mathcal{G}_{1}=\sigma\left\{\left|X_{1}(u)\right|,\left|X_{2}(u)\right|, u \geq 0\right\} \vee \sigma\left\{\operatorname{sign} X_{1}(1)\right\}, \\
& \mathcal{G}_{2}=\sigma\left\{\left|X_{1}(u)\right|,\left|X_{2}(u)\right|, u \geq 0\right\} \vee \sigma\left\{\operatorname{sign} X_{1}(1), \operatorname{sign} X_{2}(1)\right\} .
\end{aligned}
$$

Then $\mathcal{G}_{1}=\mathcal{G}_{2}$. In other words, for such Brownian motions $X_{1}$ and $X_{2}$, the observation over time of their absolute values and the sign of one of them at any given time determines the sign of the other at the same time.

Proof. Recall that the quadratic variation $\langle X\rangle$ of a diffusion $(X(u), u \geq 0)$ is given by

$$
\langle X\rangle_{u}=\lim _{n \rightarrow \infty} \sum_{k=1}^{\left[2^{n} u\right]}\left(X\left(k 2^{-n}\right)-X\left((k-1) 2^{-n}\right)\right)^{2} \quad \text { a.s. }
$$

The diffusion $X$ can be replaced by $|X|$ to define $\langle|X|\rangle$, and it is well-known that $\langle|X|\rangle_{u}=\langle X\rangle_{u}$, for all $u \geq 0$, a.s. In particular, $\langle X\rangle_{u}$ is $\sigma(|X|)$-measurable.

Clearly, the $\sigma$-fields $\mathcal{G}_{1}$ and $\mathcal{G}_{2}$ satisfy $\mathcal{G}_{1} \subset \mathcal{G}_{2}$, and recall, as mentioned in the Introduction, that these $\sigma$-fields are completed by $P$-null sets. For the converse inclusion, we show that $\operatorname{sign} X_{2}(1)$ is $\mathcal{G}_{1}$-measurable. Set $Y(u)=\left|X_{1}(u)\right|+\left|X_{2}(u)\right|$, so that the process $(Y(u), u \geq 0)$ is $\mathcal{G}_{1}$-measurable. Clearly,

$$
Y(u)= \begin{cases}\left|2 a B_{1}(u)+b B_{2}(u)\right| & \text { if } X_{1}(u) \cdot X_{2}(u) \geq 0 \\ \left|b B_{2}(u)\right| & \text { if } X_{1}(u) \cdot X_{2}(u)<0\end{cases}
$$

It follows that

$$
\frac{d\langle Y\rangle_{u}}{d u}= \begin{cases}4 a^{2}+b^{2} & \text { if } X_{1}(u) \cdot X_{2}(u)>0 \\ b^{2} & \text { if } X_{1}(u) \cdot X_{2}(u)<0\end{cases}
$$

Therefore, writing equality between sets that differ only by a null set,

$$
\begin{aligned}
\left\{X_{2}(1)>0\right\}= & \left\{\left.\frac{d\langle Y\rangle_{u}}{d u}\right|_{u=1}=4 a^{2}+b^{2}, X_{1}(1)>0\right\} \\
& \cup\left\{\left.\frac{d\langle Y\rangle_{u}}{d u}\right|_{u=1}=b^{2}, X_{1}(1)<0\right\} \\
\in & \mathcal{G}_{1} .
\end{aligned}
$$

The lemma is proved. 
Proof of Theorem 18. Let

$$
\mathcal{H}_{1}=\sigma\left\{|W(s)|, s \in \mathbb{R}_{+}^{2}\right\} \vee \sigma\{\operatorname{sign} W(1,1)\}
$$

and

$$
\mathcal{H}_{2}=\sigma\left\{W(s), s \in \mathbb{R}_{+}^{2}\right\} .
$$

Clearly, $\mathcal{H}_{1} \subset \mathcal{H}_{2}$. In order to show that $\mathcal{H}_{2} \subset \mathcal{H}_{1}$, it suffices to show that sign $W\left(s_{1}, s_{2}\right)$ is $\mathcal{H}_{1}$-measurable, for all $\left(s_{1}, s_{2}\right) \in \mathbb{R}_{+}^{2}$.

Fix $u_{2}>1, u_{2} \in \mathbb{Q}$, and set $X_{1}(u)=W(u, 1)$,

$$
X_{2}(u)=W\left(u, u_{2}\right)=B_{1}(u)+\sqrt{u_{2}-1} B_{2}(u),
$$

where $B_{1}$ and $B_{2}$ are the independent Brownian motions defined by

$$
B_{1}(u)=W(u, 1), \quad B_{2}(u)=\frac{1}{\sqrt{u_{2}-1}}\left(W\left(u, u_{2}\right)-W(u, 1)\right) .
$$

By Lemma 19] sign $\left(W\left(1, u_{2}\right)\right)$ is $\mathcal{H}_{1}$-measurable.

Fix $u_{1}>1, u_{1} \in \mathbb{Q}$, and set $\tilde{X}_{1}(v)=W(1, v)$,

$$
\tilde{X}_{2}(v)=W\left(u_{1}, v\right)=\tilde{B}_{1}(v)+\sqrt{u_{1}-1} \tilde{B}_{2}(v),
$$

where

$$
\tilde{B}_{1}(v)=W(1, v), \quad \tilde{B}_{2}(v)=\frac{1}{\sqrt{u_{1}-1}}\left(W\left(u_{1}, v\right)-W(1, v)\right) .
$$

By Lemma [19] sign $W\left(u_{1}, v\right)$ is $\mathcal{H}_{1}$-measurable because sign $W(1, v)$ is.

This shows that $\operatorname{sign} W\left(u_{1}, v\right)$ is $\mathcal{H}_{1}$-measurable, for all $u_{1} \geq 1$ and $v \geq 1$. To show that sign $W\left(u_{1}, v\right)$ is $\mathcal{H}_{1}$-measurable for $\left(u_{1}, v\right)$ in the other three quadrants relative to $(1,1)$, one can use the time-inversion properties of the Brownian sheet or continue with arguments similar to those above. This proves the theorem.

\section{REFERENCES}

[1] Dalang, R. C. and Mountford, T. S. Eccentric behaviors of the Brownian sheet along lines. Annals Probab. 30 (2002), 293-322.

[2] Dalang, R. C. and Walsh, J. B. Geography of the level sets of the Brownian sheet. Probab. Theory Related Fields 96 (1993), 153-176. MR 94h:60055

[3] Dalang, R. C. and Walsh, J. B. The structure of a Brownian bubble. Probab. Theory Related Fields 96 (1993), 475-501. MR 94j:60105

[4] Ito, K. and McKean, H. P. Diffusion processes and their sample paths. Springer-Verlag, Berlin, 1965. MR 33:8031

[5] Ikeda, N. and Watanabe, S. Stochastic Differential Equations and Diffusion Processes. NorthHolland Publishing Co., Amsterdam and New York, 1981. MR 84b:60080

[6] Knight, F. Essentials of Brownian motion and diffusion. Math. Surveys and Monographs no. 18. Amer. Math. Soc., Providence, Rhode Island, 1981. MR 82m:60098

[7] Orey, S. and Pruitt, W. Sample functions of the $N$-parameter Wiener process. Annals Probab. 1 (1973), 138-163. MR 49:11646

[8] Revuz, D. and Yor, M. Continuous martingales and Brownian motion. Grundlehren der Mathematischen Wissenschaften, Springer-Verlag, Berlin, 1991. MR 92d:60053

[9] Rogers, L. C. G. and Williams, D. Diffusions, Markov processes and martingales, vol. 2. John Wiley and Sons, New York, 1987. MR 89k:60117 
[10] Meyer, P. A. Théorie élémentaire des processus à deux indices. In: Korezlioglu, H., Mazziotto, G., Szpirglas, J. (eds). Processus aléatoires à deux indices. (Lect. Notes Math., vol. 863, pp. 1-30). Springer, Berlin, 1981. MR 83a:60059

[11] Walsh, J. An introduction to stochastic partial differential equations. In: Ecole d'Eté de Probabilités de St. Flour XIV. Lecture Notes in Mathematics 1180. Springer-Verlag, Berlin, 1986. MR 88a:60114

Département de Mathématiques, Ecole Polytechnique Fédérale, 1015 Lausanne, SWITZERLAND

E-mail address: robert.dalang@epfl.ch

Département de Mathématiques, Ecole Polytechnique Fédérale, 1015 Lausanne, Switzerland and Department of Mathematics, University of California, Los Angeles, CALIFORNia 90024

E-mail address: thomas.mountford@epfl.ch 